\title{
Sistema fotovoltaico flutuante, principais entraves e desafios de implantação no
}

\section{Brasil: uma revisão de literatura}

\author{
Floating photovoltaic system, main obstacles and implementation challenges in Brazil: a literature \\ review
}

Sistema fotovoltaico flotante, principales obstáculos y desafíos de implementación en Brasil: revisión de la literatura

\section{Resumo}

Estima-se que a população mundial será próxima a 9 bilhões em 2040. Na comparação com o consumo de 2010, para o atendimento a essa demanda será necessário um aumento de mais de $35 \%$ do fornecimento total de energia, o que demandará recursos energéticos mais diversificados. Nesse cenário, o uso de sistemas fotovoltaicos flutuantes tem progredido em muitos países, porém por se tratar de uma estratégia nova, ainda existem poucos estudos sobre estes sistemas. Entre as vantagens do uso destes dispositivos, se destacam a melhoria da eficiência de conversão, redução da evaporação da superfície da água e redução do crescimento de algas. Diante disso, este estudo teve como objetivo realizar uma revisão da literatura sobre os principais entraves e desafios de implantação dos sistemas fotovoltaicos flutuantes no Brasil. Foram utilizadas as plataformas de pesquisa acadêmica Google Acadêmico, Scientific Eletronic Library Online (SciELO) e Portal de Periódicos da Coordenação de Aperfeiçoamento de Pessoal de Nível Superior (CAPES), considerando as publicações da área de ciências ambientais e engenharias. Na presente revisão foi possível observar que o segmento de energia vem se adaptando conforme os processos de crise energética, em que novas alternativas de produção tendem a atender uma grande parcela da população e são de suma importância tanto na prevenção de apagões como na perspectiva de se ter um sistema híbrido. Constatou-se também que cada local de estudo teve suas particularidades, mas os principais entraves e desafios ainda são a viabilidade econômica em detrimento de sistemas feitos em solo e o atendimento aos atuais requisitos técnicos e de segurança necessários para instalação e operação dos sistemas.

Palavras-chave: Energia elétrica; Tecnologias; Sistema alternativos.

\begin{abstract}
It is estimated that the world population will be close to 9 billion in 2040. Compared to consumption in 2010, Attendance this demand will require a further increase, accounting for more than $35 \%$ of total energy supply. This will require more diversified energy resources. The use of floating photovoltaic systems has progressed in many countries, as it is a new strategy, there are still few studies on these systems. Among the advantages of using these devices stand out improved conversion efficiency, the reduction of evaporation from the water surface and reduction of algae growth. In this sense, this study aimed to carry out a literature review on the main obstacles and challenges of implementation in Brazil. Databases in the area of environmental sciences and engineering were used, such as: Academic Google, Scientific Electronic Library Online (SciELO) and CAPES Journal Portal. In this review, it was possible to observe that the energy sector has been adapting according to its energy crisis processes, where new production alternatives tend to supply a large portion of the population. It is of paramount importance both in preventing blackouts and in the perspective of having a hybrid system. Another important data found that, each location had its particularity, but the main obstacles and challenges are the economic viability in the detriment of systems made on the ground and attendence of all the current technical and safety requirements necessary for its installation and operation.
\end{abstract}

Keywords: Eletric energy; Technology; Alternative system. 


\begin{abstract}
Resumen
Se estima que la población mundial será cercana a los 9 mil millones en 2040. En comparación con el consumo en 2010, satisfacer esta demanda requerirá un aumento adicional, lo que representa más del 35\% del suministro total de energía. Esto requerirá recursos energéticos más diversificados. El uso de sistemas fotovoltaicos flotantes ha avanzado en muchos países, ya que es una nueva estrategia, aún existen pocos estudios sobre estos sistemas. Entre las ventajas de utilizar estos dispositivos destacan eficiencia de conversión mejorada, la reducción de la evaporación de la superficie del agua y la reducción del crecimiento de algas. En este sentido, este estudio tiene como objetivo realizar una revisión de la literatura sobre los principales obstáculos y desafíos de implementación en Brasil. Se utilizaron bases de datos en el área de ciencias ambientales e ingeniería, tales como: Google Académico, Biblioteca Electrónica Científica en Línea (SciELO) y Portal de Revistas CAPES. En esta revisión se pudo observar que el sector energético se ha ido adaptando de acuerdo a sus procesos de crisis energética, donde las nuevas alternativas de producción tienden a abastecer a una gran parte de la población. Es de suma importancia tanto en la prevención de apagones como en la perspectiva de tener un sistema híbrido. Otro dato importante encontró que cada ubicación tenía su particularidad, pero los principales factores de esta variable siguen siendo la viabilidad económica a expensas de los sistemas realizados en tierra y que cumplen con todos los requisitos técnicos y de seguridad vigentes necesarios para su instalación y funcionamiento.
\end{abstract}

Palabras clave: Energía electrica; Tecnología; Sistema alternativos.

\title{
1. Introdução
}

Embora a crise energética não seja um assunto recente, ainda é um tema de constante debate na sociedade e se constitui em um dos grandes desafios da atualidade. Alguns fatores relacionados a isso podem ser destacados, como a redução das reservas mundiais de petróleo, após a crise na década de 1970, o impacto ambiental causado pelo uso de energias não renováveis e a crescente demanda por recursos naturais. O crescimento contínuo da população e de bens de consumo tem gerado incertezas sobre o futuro energético mundial e discussões importantes no núcleo global (Cabral \& Vieira, 2012).

Além disso, estima-se que a população mundial será próxima a 9 bilhões em 2040. Na comparação com o consumo de 2010, para o atendimento a essa demanda será necessário um aumento de mais de 35\% do fornecimento total de energia. Isso exigirá recursos energéticos mais diversificados, inovações tecnológicas ambientais com maior eficiência confiável para tornar a matriz mundial sustentável (Internacional Energy Agency, 2015).

No Brasil a fonte hidráulica (consistindo em usinas hidrelétricas, pequenas centrais hidrelétricas e centrais geradoras hidrelétricas) gerou 63,8\% da eletricidade em 2020, enquanto outras fontes de energia 36,2\%. Dentre essas fontes, o destaque é a geração de energia termoelétrica, eólica, gás natural e biomassa. No entanto, a mudança mais significativa verificada entre 2019 e 2020 é que a geração fotovoltaica aumentou 61,1\%, enquanto o uso de termoelétricas diminuiu 22,1\% (Empresa de Pesquisa Energética, 2021).

Nesse sentido, é fundamental complementar a matriz energética com outras fontes de energia, para reduzir os impactos ambientais gerados pela implantação e operação das fontes convencionais, e garantindo que a demanda seja atendida em condições seguras e adequadas (Tolmasquim, 2016). Dentre as diversas tecnologias geradoras de energia renovável, podemos citar a energia eólica, os sistemas fotovoltaicos, as usinas de incineração de resíduos e biomassa e as pequenas centrais hidroelétricas com as possíveis alternativas para a geração distribuída de energia (Dematte et al., 2016).

A tecnologia fotovoltaica vem sendo amplamente utilizada no Brasil, pois o país tem grande potencial para desenvolver esse recurso. No entanto, uma das dificuldades encontradas na instalação de projetos fotovoltaicos é a ocupação do espaço urbano, nesse sentido os projetos de painéis fotovoltaicos flutuantes têm sido explorados (Silva \& Souza, 2017).

Como aponta Choi (2014), o sistema fotovoltaico flutuante é composto por: a) sistema de flutuação: abrange uma armação flutuante, com uma estrutura com flutuador, permitindo a instalação do módulo fotovoltaico; b) sistema de ancoragem: possibilita ajustar a flutuação do nível da água mantendo a posição do sistema na direção norte (hemisfério sul); c) sistema fotovoltaico: são os equipamentos de geração solar fotovoltaica, inseridos sobre o sistema de flutuação; e d) cabos de energia: responsáveis pela transmissão da energia. 
Uma vez que o conceito de energia fotovoltaica flutuante só surgiu recentemente, todas as vantagens e limitações deste sistema não foram claramente determinadas devido as peculiaridades de cada local de implantação (Costa, 2017). De forma geral, entre as vantagens do uso destes dispositivos se destacam a redução da evaporação da superfície da água, redução do crescimento de algas e, devido ao efeito de resfriamento dos módulos fotovoltaicos, ocorre a melhoraria da eficiência de conversão (Lopes \& Junior, 2016). Em comparação com as instalações tradicionais, os sistemas fotovoltaicos flutuantes ainda são considerados de risco relativamente alto pelos investidores e pela comunidade científica. (Rebelo, 2021). As fontes energias renováveis alternativas, como a geração fotovoltaica flutuante, apresentam diversas vantagens, mas ainda trazem desafios que precisam ser mais bem compreendidos e superados (Borda \& Novak, 2018).

Nesse sentido, este estudo tem como objetivo realizar uma revisão da literatura sobre os principais entraves e desafios de implantação no Brasil da geração de energia fotovoltaica flutuante em corpos d'água.

\section{Metodologia}

Este estudo é uma revisão descritiva exploratória da literatura com estratégia qualitativa, que apresenta com detalhes os entraves e desafios do uso da energia fotovoltaica flutuante a partir da consulta em estudos já publicados (Rother, 2007).

A pesquisa foi realizada de acordo com as seguintes etapas: identificação dos sujeitos, elaboração dos critérios de inclusão e exclusão, elaboração e seleção das pesquisas, análise e avaliação dos resultados.

Foram selecionadas publicações no formato de artigos científicos, monografias, dissertações e trabalhos apresentados em eventos técnicos e científicos disponíveis nas plataformas de pesquisa acadêmicas para a área de ciências ambientais e engenharias: Google Acadêmico, Scientific Eletronic Library Online (SciELO) e Portal de Periódicos da Coordenação de Aperfeiçoamento de Pessoal de Nível Superior (CAPES), utilizando os descritores: sistema fotovoltaico flutuante; desafios e implantação.

Como critérios de inclusão, foram selecionados estudo sobre o tema selecionado, os quais foram publicados em língua portuguesa entre os anos de 2010 e 2021. Os critérios de exclusão são estudos destoantes para o assunto, publicações anteriores a 2010 e trabalhos incompletos.

Após uma análise detalhada das publicações envolvendo os filtros de pesquisa, foram utilizados 10 estudos que correspondiam aos tópicos desta revisão de literatura.

\section{Resultados e Discussão}

No Quadro 1 apresenta-se os 10 estudos realizados com diversos projetos de instalação de usinas fotovoltaicas flutuantes e que foram analisados para verificar os entraves e desafios desse tipo de sistema no Brasil. 
Quadro 1 - Resultados dos estudos entre os anos 2016 a 2021.

\begin{tabular}{|c|c|c|c|c|c|c|}
\hline Título do Estudo & Autor/Ano & Local & $\begin{array}{l}\text { Geração de } \\
\text { Energia/ } \\
\text { Área }\end{array}$ & Objetivo & Entraves e Desafios & $\begin{array}{l}\text { Resultado/ } \\
\text { Conclusão }\end{array}$ \\
\hline $\begin{array}{c}\text { Usinas fotovoltaicas } \\
\text { flutuantes como } \\
\text { alternativa de geração } \\
\text { de energia e redução de } \\
\text { evaporação no Sistema } \\
\text { Cantareira de } \\
\text { abastecimento de São } \\
\text { Paulo } \\
\end{array}$ & DIAS (2021) & $\begin{array}{c}\text { SÃO PAULO- } \\
\text { SP }\end{array}$ & $1 \mathrm{MWp} / 5.925 \mathrm{~m}^{2}$ & $\begin{array}{c}\text { Realizar um estudo técnico e econômico } \\
\text { da instalação de usinas fotovoltaicas } \\
\text { flutuantes nos mananciais que abastecem } \\
\text { o Sistema de Cantareira, estimando } \\
\text { quanto pode ser gerado de energia e a } \\
\text { quantidade de água evaporada que pode } \\
\text { ser evitada. }\end{array}$ & $\begin{array}{c}\text { Venda de energia gerada } \\
\text { pela usina fotovoltaica } \\
\text { flutuante em leilões ou } \\
\text { contratação livre e } \\
\text { avaliações técnicas, } \\
\text { econômicas, sociais e } \\
\text { ambientais. }\end{array}$ & $\begin{array}{c}\text { Diante do exposto, o estudo } \\
\text { estimou uma redução na taxa de } \\
\text { evaporação das represas e o } \\
\text { aumento na geração extra de } \\
\text { energia gerada a partir das } \\
\text { usinas fotovoltaicas flutuantes. }\end{array}$ \\
\hline $\begin{array}{c}\text { Plataforma fotovoltaica } \\
\text { flutuante de Sobradinho } \\
\text { (BA) - desafios e } \\
\text { estratégias de } \\
\text { implantação }\end{array}$ & $\begin{array}{c}\text { RODRI.GU } \\
\text { ES et al, } \\
(2020)\end{array}$ & $\begin{array}{c}\text { SOBRADINHO- } \\
\text { BA }\end{array}$ & $\begin{array}{l}2,5 \mathrm{MWp} / \\
27.000 \mathrm{~m}^{2}\end{array}$ & $\begin{array}{c}\text { Estudar os fatores relacionados à } \\
\text { interação entre a tecnologia solar } \\
\text { flutuante e a operação de usinas } \\
\text { hidrelétricas. }\end{array}$ & $\begin{array}{c}\text { Alto índice de } \mathrm{pH} \text { na água } \\
\text { e outros elementos } \\
\text { químicos, atender a todos } \\
\text { os atuais requisitos } \\
\text { técnicos e de segurança } \\
\text { necessários. }\end{array}$ & $\begin{array}{c}\text { A tecnologia fotovoltaica } \\
\text { flutuante instalada no } \\
\text { reservatório com a operação } \\
\text { conjunta de usinas hidrelétricas } \\
\text { é uma alternativa em potencial } \\
\text { para compensar as } \\
\text { vulnerabilidades da energia } \\
\text { hidrelétrica e otimizar a } \\
\text { produção de energia dos dois } \\
\text { sistemas. }\end{array}$ \\
\hline $\begin{array}{l}\text { Estudo de viabilidade } \\
\text { técnica de usina solar } \\
\text { fotovoltaica flutuante } \\
\text { para suprir consumo da } \\
\text { Universidade de } \\
\text { Brasília }\end{array}$ & $\begin{array}{c}\text { SILVA \& } \\
\text { SHAYANE, } \\
(2020)\end{array}$ & BRASÍLIA-DF & $\begin{array}{c}15,3 \\
\mathrm{MWp} / 84.300 \mathrm{~m}^{2}\end{array}$ & $\begin{array}{c}\text { Estudar duas modalidades de aplicação } \\
\text { da tecnologia fotovoltaica, isto é, sistema } \\
\text { de grande porte e sistema flutuante, } \\
\text { através de um estudo de viabilidade. }\end{array}$ & $\begin{array}{c}\text { Produção anual de } \\
\text { energia igual ao consumo } \\
\text { anual de energia da } \\
\text { universidade }\end{array}$ & \begin{tabular}{|c|} 
O estudo de viabilidade \\
realizado determinou que a \\
implantação do sistema de \\
geração de energia fotovoltaica \\
na UnB é tecnicamente viável e \\
a produção anual de energia do \\
sistema é igual ao consumo \\
anual de energia da \\
Universidade de Brasília. \\
\end{tabular} \\
\hline $\begin{array}{l}\text { Análise de desempenho } \\
\text { de sistemas } \\
\text { fotovoltaicos } \\
\text { experimentais no } \\
\text { reservatório da UHE de } \\
\text { Santa Clara }\end{array}$ & $\begin{array}{c}\text { FRANCO et } \\
\text { al., (2020) }\end{array}$ & PINHÃO-PR & $\begin{array}{l}100,74 \mathrm{kWp} / \\
\text { NÃO } \\
\text { DISPONÍVEL }\end{array}$ & $\begin{array}{l}\text { Comparar a produção real entre um } \\
\text { sistema fotovoltaico flutuante e três } \\
\text { sistemas fotovoltaicos em solo. }\end{array}$ & $\begin{array}{c}\text { Eventos climáticos e } \\
\text { viabilidade econômica. }\end{array}$ & $\begin{array}{c}\text { Como resultado, a energia } \\
\text { gerada durante o estudo foi } \\
\text { inferior a } 56 \% \text { do valor médio } \\
\text { observado nos últimos três } \\
\text { meses desde a sua ligação à } \\
\text { rede. Para este experimento, } \\
\text { RETScreen mostrou que durante } \\
\text { os } 25 \text { anos de escopo do projeto, } \\
\text { a produção anual de energia para } \\
\text { a rede foi de aproximadamente } \\
\text { 131 MWh. Porém, devido ao um } \\
\text { incidente, foi obtida uma } \\
\text { potência de aproximadamente } \\
80 \mathrm{MWh} \text {. } \\
\end{array}$ \\
\hline $\begin{array}{l}\text { Estudo sobre usinas } \\
\text { solares flutuantes em } \\
\text { reservatórios de água } \\
\text { no Brasil: Estudo de } \\
\text { caso no Rio Mossoró }\end{array}$ & $\begin{array}{c}\text { CARVALH } \\
\text { O (2020). }\end{array}$ & MOSSORÓ-RN & $\begin{array}{c}108524,26 \mathrm{kWh} / \\
4767,42 \mathrm{~m}^{2}\end{array}$ & $\begin{array}{c}\text { Apresentar os conceitos básicos dos } \\
\text { sistemas solares tradicionais e os } \\
\text { componentes básicos dos sistemas } \\
\text { solares flutuantes. Por fim, serão } \\
\text { apresentadas as dimensões da usina solar } \\
\text { flutuante planejada para o município de } \\
\text { Mossoró-RN, que poderá ser instalada na } \\
\text { superfície do rio Mossoró. }\end{array}$ & $\begin{array}{l}\text { Sinuosidade da área de } \\
\text { instalação. }\end{array}$ & $\begin{array}{c}\text { Observou-se o aumento do } \\
\text { potencial energético, agregando } \\
\text { energia que pode atender às } \\
\text { necessidades de mais de } 700 \\
\text { casas em seu sistema básico. } \\
\text { Deve-se ressaltar que o } \\
\text { dimensionamento utiliza apenas } \\
\text { uma pequena parte de toda a } \\
\text { área útil onde podem ser } \\
\text { instalados equipamentos } \\
\text { flutuantes. Entende-se também } \\
\text { que a construção de usinas } \\
\text { solares flutuantes não se limita } \\
\text { ao caso de usinas hidrelétricas, } \\
\text { mas inclui qualquer tipo de caso } \\
\text { que tenha uma área de cobertura } \\
\text { hídrica razoável e possa ser } \\
\text { instalado e mantido. }\end{array}$ \\
\hline $\begin{array}{l}\text { Estudo de caso da } \\
\text { utilização de energia } \\
\text { fotovoltaica flutuante } \\
\text { no reservatório de } \\
\text { Passo Real }\end{array}$ & $\begin{array}{l}\text { CEZAR } \\
\text { (2019). }\end{array}$ & $\begin{array}{c}\text { SALTO } \\
\text { DO JACUÍ-RS }\end{array}$ & $\begin{array}{c}4,95 \mathrm{MWp} / 1,45 \\
\text { ha }\end{array}$ & $\begin{array}{c}\text { Avaliar o potencial de um } \\
\text { empreendimento no reservatório, tais } \\
\text { como levantamento de dados da usina } \\
\text { hidrelétrica, pesquisa de radiação, } \\
\text { dimensionamento da planta, geração de } \\
\text { energia, possíveis materiais e } \\
\text { equipamentos, possíveis impactos } \\
\text { ambientais e prováveis localizações. }\end{array}$ & \begin{tabular}{|} 
Atenuar riscos e impactos \\
para a fauna, análise \\
periódica da qualidade da \\
água no local, \\
acompanhamento de \\
especialistas para \\
identificação e correção \\
de problemas.
\end{tabular} & $\begin{array}{c}\text { O resultado constatou que a } \\
\text { previsão de energia gerada pelo } \\
\text { sistema de cada usina } \\
\text { fotovoltaica flutuante aumente } \\
\text { levemente em } 0,26 \% \text { no } \\
\text { incremento de energia gerada. }\end{array}$ \\
\hline
\end{tabular}




\begin{tabular}{|c|c|c|c|c|c|c|}
\hline $\begin{array}{c}\text { Análise técnico- } \\
\text { econômica de usina } \\
\text { Fotovoltaica flutuante } \\
\text { no Lago } \\
\text { Paranoá para redução } \\
\text { no gasto com } \\
\text { Consumo da } \\
\text { Universidade de } \\
\text { Brasília } \\
\end{array}$ & $\begin{array}{l}\text { RIOS } \\
(2019) .\end{array}$ & BRASILIA-DF & $\begin{array}{c}501,6 \mathrm{~kW} / \\
148.516,25 \mathrm{~m}^{2}\end{array}$ & $\begin{array}{l}\text { Neste trabalho, foram apresentadas as } \\
\text { análises técnicas e econômicas de dois } \\
\text { tipos de usinas, solo e flutuante, para } \\
\text { serem instaladas com o objetivo de } \\
\text { compensar os custos com consumo da } \\
\text { Universidade de Brasília - Campus } \\
\text { Darcy Ribeiro. }\end{array}$ & $\begin{array}{c}\text { Valores maiores para a } \\
\text { instalação dos sistemas } \\
\text { fotovoltaicos flutuantes } \\
\text { em detrimento dos } \\
\text { sistemas em solo. }\end{array}$ & $\begin{array}{c}\text { A usina flutuante é } \\
\text { economicamente viável e, apesar } \\
\text { disso, a usina } \\
\text { em solo é um melhor } \\
\text { investimento. }\end{array}$ \\
\hline $\begin{array}{c}\text { Estudo de uma usina } \\
\text { solar fotovoltaica } \\
\text { flutuante em um lago } \\
\text { de hidrelétrica - UHE } \\
\text { Tucuruí }\end{array}$ & $\begin{array}{l}\text { SOUSA } \\
(2017) .\end{array}$ & TUCURUÍPA & $\begin{array}{c}1 \mathrm{MWp} / 9.660,6 \\
\mathrm{~m}^{2}\end{array}$ & $\begin{array}{c}\text { Reunir as bases teóricas necessárias para } \\
\text { melhor compreender os temas } \\
\text { relacionados: a implantação de usinas } \\
\text { solares fotovoltaicas em lagos de } \\
\text { reservatórios de energia hidrelétrica para } \\
\text { aumentar a diversidade energética do } \\
\text { país. }\end{array}$ & \begin{tabular}{|c|} 
Validar algumas \\
certificações de módulos \\
fotovoltaicos e inversores \\
solares de forma a \\
selecionar produtos de \\
qualidade comprovada no \\
projeto e fabricação de \\
um sistema de flutuação \\
próprio.
\end{tabular} & $\begin{array}{c}\text { O estudo mostrou que uma usina } \\
\text { flutuante de } 1 \mathrm{MW} \text { pode ser } \\
\text { obtida em uma área de } \\
\text { aproximadamente } 10.000 \text { metros } \\
\text { quadrados, e } 3.712 \text { módulos de } \\
270 \mathrm{~W} .\end{array}$ \\
\hline $\begin{array}{c}\text { Estimativa de geração } \\
\text { de energia através de } \\
\text { um sistema } \\
\text { fotovoltaico: } \\
\text { implicações para um } \\
\text { sistema flutuante no } \\
\text { Lago Bolonha, Belém- } \\
\text { Pará. }\end{array}$ & $\begin{array}{l}\text { SILVA \& } \\
\text { SOUZA } \\
(2017)\end{array}$ & BELÉM-PA & $\begin{array}{c}38.012 \mathrm{kWh} / 183 \\
\mathrm{~m}^{2}\end{array}$ & $\begin{array}{c}\text { Estimar a quantidade de energia elétrica } \\
\text { que será gerada com a aplicação do } \\
\text { projeto fotovoltaico usando o software } \\
\text { SAM versão 2015.1.30. }\end{array}$ & $\begin{array}{c}\text { Realizar estimativa } \\
\text { baseada nos dados de um } \\
\text { sistema em terra e perdas } \\
\text { como: sombreamento, } \\
\text { poeiras, perda nos } \\
\text { módulos solares, conexão } \\
\text { e fiações. }\end{array}$ & $\begin{array}{c}\text { Demonstraram um potencial uso } \\
\text { dessa tecnologia para uso de } \\
\text { energia na própria estação de } \\
\text { tratamento de água, no Parque } \\
\text { do Utinga e até mesmo nas } \\
\text { comunidades do entorno do } \\
\text { parque. }\end{array}$ \\
\hline $\begin{array}{l}\text { Sistemas Fotovoltaicos } \\
\text { Flutuantes: análise do } \\
\text { tema e estudo de caso } \\
\text { para o lago da UNIFEI }\end{array}$ & $\begin{array}{l}\text { LOPES \& } \\
\text { JUNIOR } \\
(2016)\end{array}$ & ITAJUBÁ-MG & $\begin{array}{c}206 \mathrm{KWp} / 3.200 \\
\mathrm{~m}^{2}\end{array}$ & $\begin{array}{c}\text { Analisar aspectos relacionados à } \\
\text { instalação de sistemas solares } \\
\text { fotovoltaicos na água, como lagos, mar e } \\
\text { barragens. }\end{array}$ & $\begin{array}{c}\text { Economia na receita com } \\
\text { energia elétrica e status } \\
\text { para instituição produtora } \\
\text { de energia. }\end{array}$ & \begin{tabular}{|c|} 
O estudo demonstrou que em \\
caso do sistema solar \\
fotovoltaico flutuante for \\
instalado, estima-se que seria \\
capaz de abastecer cerca de $15 \%$ \\
da procura total de eletricidade \\
em um ano. \\
\end{tabular} \\
\hline
\end{tabular}

Fonte: Autores.

No estudo realizado pelo Dias (2021), estimou-se que a instalação da usina fotovoltaica flutuante no sistema Cantareira poderia atender até 99.500 pessoas com geração de energia anual entre de 7,2 TWh e 11,4 TWh. Segundo Rodrigues et al., (2020), a tecnologia fotovoltaica flutuante instalada em extenso espelho de água como reservatórios para abastecimento e de usinas hidrelétricas para operação combinada e complementar à hidroeletricidade representa alternativa com grande potencial para compensar os pontos de vulnerabilidade da geração hidroelétrica e otimizar a produção de energia de ambos os sistemas.

Na pesquisa realizada por Silva e Shayane (2020), determina-se que é tecnicamente viável implantar o sistema de geração de energia fotovoltaica na UnB. O resultado da simulação do sistema em um ano meteorológico típico foi uma geração de energia anual de 25.843,0 MWh, portanto, atinge e excede o objetivo inicial de geração de 24.933,51 MWh/ano para igualar o consumo anual médio de energia da Universidade de Brasília. No estudo de caso realizado por Lopes e Junior (2016), o lago da UNIFEI poderia receber a instalação de 792 módulos fotovoltaicos, com uma potência nominal de $206 \mathrm{kWp}$, com isso seria capaz de fornecer por volta de $15 \%$ da demanda total de eletricidade anual da instituição.

Franco (2020), a energia gerada durante o estudo foi inferior a 56\% do valor médio observado nos últimos três meses desde a sua ligação à rede devido ao um evento de fortes chuvas na região em que ocorreu o desligamento de grande parte dos módulos dos painéis flutuantes. No experimento durante os 25 anos de escopo do projeto, a produção anual de energia para a rede foi de aproximadamente $131 \mathrm{MWh}$, porém devido ao incidente foi obtida uma potência de aproximadamente $80 \mathrm{MWh}$. Os resultados evidenciam que a ocorrência de acidentes e desastres naturais, especialmente os relacionados a eventos meteorológicos devem ser considerados e podem ser considerados entraves.

No estudo de base de Carvalho (2020), aborda-se uma possível implementação de uma usina solar fotovoltaica flutuante na cidade de Mossoró - RN, para uma determinada área de 4767,42 m² e com 2.000 painéis fotovoltaicos em operação. Nestas condições a geração mensal de energia seria de aproximadamente $108.524,26 \mathrm{kWh}$, suficiente para atender às necessidades de mais de 730 residências. 
Cezar (2019), mostrou-se uma expectativa de incremento marginal de 0,26\% na energia gerada pelo sistema combinado UHE e módulos de usina fotovoltaica flutuante. Deve-se ressaltar que esse incremento foi alcançado com o uso de apenas uma pequena fração da área disponível no lago (1,45 ha), o que evidencia que a disponibilidade de área adequada para instalação do sistema flutuante pode ser um entrave para viabilidade do sistema. Nesse sentido, conforme a pesquisa realizada por Sousa (2017), em que a intenção do estudo é compreender melhor a instalação de usinas solares fotovoltaicas em lagos hidrelétricos e descrever os possíveis benefícios de sua utilização e seu potencial de impacto, se houver disponibilidade de área os ganhos em termos de aumento da capacidade de geração são significativos. No estudo, a implantação do sistema flutuante pode aumentar a capacidade da usina já instalada em até $40 \%$, ocupando apenas $1,16 \%$ do reservatório ou $33 \mathrm{~km}^{2}$, sem gerar novos investimentos no sistema elétrico, com isso, é possível adicionar 3.414 mw de energia elétrica ao SIN (Sistema Interligado Nacional).

Segundo a análise realizada por Rios (2019), foi feita a análise de ganho do sistema flutuante ao se alterar a temperatura e a perda por incrustações, com o resultado obtido a geração de energia pode chegar a $81.077 \mathrm{kWh}$ mensais, o ganho do sistema flutuante em relação ao em solo foi de 4,8\%. Na análise de viabilidade econômica, constatou-se que o investimento inicial na planta terrestre é de $\mathrm{R} \$ 47.732 .256,00$ e o investimento inicial na planta flutuante é maior ( $\mathrm{R} \$ 52.542 .600,00)$, com isso observase que a usina flutuante é economicamente viável, apesar disso, a usina em solo ainda é um melhor investimento do ponto de vista econômico (Leite et al., 2021; Silva et al., 2020), sendo que os custos do sistema flutuante pode ser representar uma dificuldade para implantação dos mesmos.

Conformo trabalho do autor Silva e Sousa (2017), a pesquisa fornece uma revisão da literatura das Usinas Fotovoltaicas Flutuantes e tenta enfatizar e destacar a aplicação teórica dessa nova concepção no Lago Bolonha em Belém-PA, usando o software SAM. A modelagem empírica estimou que a produção anual de 112250 painéis seria de $38.012 \mathrm{kWh}$, o que mostra que a tecnologia tem grande potencial para uso de energia em larga e pequena escala.

\section{Conclusão}

No presente estudo foi possível observar que o segmento de energia vem se adaptando conforme seus processos de crise energética, onde novas alternativas de produção tendem a suprir uma grande parcela da população o que é de suma importância tanto para a prevenção de apagões como na perspectiva de se ter um sistema híbrido.

Pode se observar que a busca por sistemas fotovoltaicos flutuantes é crescente no Brasil, onde a região Nordeste é a que apresenta o maior potencial devido a maiores taxas de irradiação solar durante todo o ano e baixa influência de massas úmidas e frias vindo do sul.

Em relação aos entraves e desafios constatou-se que em cada local teve sua particularidade, porém se destacam a viabilidade econômica em detrimento de sistemas em solo e atendimento aos atuais requisitos técnicos e de segurança necessários para sua instalação e operação.

Nesse sentido, os estudos sobre os entraves e desafios dos sistemas fotovoltaicos flutuantes no Brasil são de grande relevância para identificar e mensurar as adversidades deste novo segmento e como forma de contribuir para a expansão do uso destes sistemas como fonte de energia renovável. Recomendam-se novos estudos que abordem o tema evidenciando a viabilidade econômica e técnica, assim como o avanço da tecnologia.

\section{Referências}

Borda, R. A., \& Novak, L. H. (2020). Sistemas Fotovoltaicos Flutuantes: aspectos positivos e desafios. In: Congresso Brasileiro De Energia Solar, 8, FortalezaCE, 2020. Anais...CBENS, 2020.

Cabral, I., \& Vieira, R. Viabilidade econômica x viabilidade ambiental do uso de energia fotovoltaica no caso brasileiro: uma abordagem no período recente. In. III Congresso Brasileiro De Gestão Ambiental, 2012. Anais..., CBGA2012. 
Carvalho, T. F. (2020). Estudo sobre usinas solares flutuantes em reservatórios de água no Brasil: estudo de caso no rio Mossoró. Trabalho de Conclusão de Curso (Graduação em Engenharia Elétrica), Centro de Engenharias, Universidade Federal Rural do Semi-Árido, Mossoró, Rio Grande do Norte. 11p.

Cezar, P. H. D. P. (2019). Estudo De Caso Da Utilização De Energia Fotovoltaica Flutuante No Reservatório De Passo Real. Trabalho de Conclusão de Curso (Graduação em Engenharia Elétrica), Universidade Federal do Rio Grande do Sul, Porto Alegre-RS. 53 p.

Choi, Y. K. (2014). A study on power generation analysis of floating PV system considering environmental impact. International journal of software engineering and its applications, 8(1), 75-84.

Costa, S. G. (2017). Impactes ambientais de sistemas fotovoltaicos flutuantes. Dissertação de mestrado (Mestrado Integrado em Engenharia da Energia e Ambiente), Universidade de Lisboa, Liboa-PT. 146 p.

Dematte, R. D. et al. (2016). Análise de Eficiência de Fontes de Energia Renováveis. In: Brazilian Techonology Symposium, Campinas-SP. Anais..., BTSYM, 2016.

Dias, C. M. (2021). Usinas Fotovoltaicas Flutuantes Como Alternativa de Geração de Energia e Redução de Evaporação no Sistema Cantareira de Abastecimento De São Paulo. Trabalho de Conclusão (Graduação em Engenharia Mecânica), Universidade Federal do Rio de Janeiro, Rio de Janeiro-RJ. 120 p.

Empresa de Pesquisa Energética. (2021). Anuário Estatístico De Energia Elétrica 2021. https://www.epe.gov.br/sites-pt/publicacoes-dadosabertos/publicacoes/PublicacoesArquivos/publicacao-160/topico-168/Anu\%C3\%A1rio_2021.pdf

Franco, P. R. et al. (2020). Análise de desempenho de sistemas fotovoltaicos experimentais no reservatório da UHE de Santa Clara. Revista Aberta de Energia do Brasil, 33, 33-43.

International Energy Agency. (2015). Key Word Energy Statistics. Paris: IEA. https://iea.blob.core.windows.net/assets/52f66a88-0b63-4ad2-94a529d36e864b82/KeyWorldEnergyStatistics2021.pdf

Leite, M. D. S. et al. (2021) Technical and Financial Feasibility of implementing a photovoltaic system in a private Higher Education Institution in Cajazeiras PB. Research, Society and Development, 10(6), 1-13.

Lopes, M. M., \& Junior, P. A. S. (2016). Sistemas fotovoltaicos flutuantes: análise do tema e estudo de caso para o lago da UNIFEI. In: $X$ Congresso Brasileiro De Planejamento Energético, 10, Gramado-RS. Anais... X CBPE, 2016.

Rebelo, R. A. R. (2021). Estudo do potencial de sistemas fotovoltaicos flutuantes em massas de água doce e marinhas. Dissertação (Mestrado em Engenharia da Energia Solar), Escola de Ciências e Tecnologias, Universidade de Évora, Évora-PT. 100 p.

Rios, C. A. (2019). Análise Técnico-Econômica de Usina Fotovoltaica Flutuante no Lago Paranoá Para Redução no Gasto Com Consumo da Universidade de Brasília. Trabalho de Conclusão de Curso (Engenharia Elétrica), Departamento de Engenharia Elétrica, Universidade de Brasília, Brasília-DF. 114 p.

Rodrigues, P. S. F. et al. (2020). Plataforma Fotovoltaica Flutuante de Sobradinho (BA) - Desafios e Estratégias de Implantação. In: CONGRESSO BRASILEIRO DE ENERGIA SOLAR, 8, Fortaleza-CE. Anais...XIII CBENS.

Rother, E. T. (2017). Revisão sistemática X revisão narrativa. Acta Paulista de Enfermagem, 20(2), 5-6.

Silva, G. D. P., \& Souza, M. J. R. (2017). Estimativa de Geração de Energia através de um Sistema Fotovoltaico: Implicações para um Sistema Flutuante no Lago Bolonha, Belém-Pará. Revista Brasileira de Energias Renováveis, 6(2), 149-164.

Silva, P. H. B., \& Shayani, R. A. (2020). Estudo de viabilidade técnica de usina solar fotovoltaica flutuante para suprir consumo da Universidade de Brasília. In: Congresso Brasileiro De Energia Solar, 8, Fortaleza-CE., Anais...XIII CBENS.

Silva, D. V. et al., (2020). Study on the economic viability of a photovoltaic plant in a higher education institution in the semi-arid region of Paraíba. Research, Society and Development, 9 (11), 1-14.

Sousa, A. C. (2017). Estudo de uma Usina Solar Fotovoltaica Flutuante em um Lago de Hidrelétrica - UHE Tucuruí. Trabalho de Conclusão de Curso (Graduação em Engenharia de Energia), Universidade de Brasília, Brasília-DF. 58 p.

Tolmasquim, M. T. (2016). Energia renovável: hidráulica, biomassa, eólica, solar, oceânica. Empresa de Pesquisa Energética. 452 p. 